\title{
Strongly Summable Double Sequence Spaces in $n$-Normed Spaces Defined by Ideal Convergence and an Orlicz Function
}

\author{
AYHAN EsI \\ Adiyaman University, Science and Art Faculty, Department of Mathematics, 02040, \\ Adiyaman, Turkey \\ e-mail : aesi23@hotmail.com
}

Abstract. In this paper we introduce some new double sequence spaces via ideal convergence and an Orlicz function in $n$-normed spaces and examine some properties of the resulting spaces.

\section{Introduction}

Let $X$ be a non-empty set, then a family of sets $I \subset 2^{X}$ (the class of all subsets of $X$ ) is called an ideal if and only if for each $A, B \in I$, we have $A \cup B \in I$ and for each $A \in I$ and each $B \subset A$, we have $B \in I$. A non-empty family of sets $F \subset 2^{X}$ is a filter on $X$ if and only if $\varnothing \notin F$, for each $A, B \in F$, we have $A \cap B \in F$ and each $A \in F$ and each $A \subset B$, we have $B \in F$. An ideal $I$ is called non-trivial ideal if $I \neq \varnothing$ and $X \notin I$. Clearly $I \subset 2^{X}$ is a non-trivial ideal if and only if $F=F(I)=\{X / A: A \in I\}$ is a filter on $X$. A non-trivial ideal $I \subset 2^{X}$ is called admissible if and only if $\{\{x\}: x \in X\} \subset I$. Further details on ideals of $2^{X}$ can be found in Kostyrko, et.al [5]. The notion was further investigated by Salat et.al [13], Tripathy and Hazarika [21, 23, 15, 29], Tripathy and Mahanta [27] and others.

The notion of double sequences has been investigated from different aspect by Tripathy and Dutta [25], Tripathy and Sarma [22, 24, 30] and many others in recent years.

By the convergence of a double sequence we mean the convergence on the Pringsheim sense that is, a double sequence $x=\left(x_{k, l}\right)$ has Pringsheim limit $L$ (denoted by $P-\lim x=L)$ provided that given $\varepsilon>0$ there exists $n \in \mathbb{N}$ such that $\left|x_{k, l}-L\right|<\varepsilon$ whenever $k, l>n$ Pringsheim [10]. We shall write more briefly as " $P$-convergent".

The double sequence $x=\left(x_{k, l}\right)$ is bounded if there exists a positive number $M$ such that $\left|x_{k, l}\right|<M$ for all $k$ and $l$. Let $l_{\infty}^{2}$ the space of all bounded double such that

$$
\left\|x_{k, l}\right\|_{(\infty, 2)}=\sup _{k, l}\left|x_{k, l}\right|<\infty .
$$

Received January 9, 2011; revised September 14, 2011; accepted November 24, 2011. 2010 Mathematics Subject Classification: Primary 40A99; Secondary 40A05.

Key words and phrases: P-convergent, n-normed space, Orlicz function. 
Recall Krasnoselski and Rutickii [6] that an Orlicz function $M$ is continuous, convex, nondecreasing function define for $x>0$ such that $M(0)=0$ and $M(x)>0$. If convexity of Orlicz function is replaced by $M(x+y) \leq M(x)+M(y)$ then this function is called the modulus function and characterized by Nakano [9]. It was further investigated from sequence space point of view by Ruckle [12], Tripathy and Chandra [28] and many others. An Orlicz function $M$ is said to satisfy $\Delta_{2}-$ condition for all values $u$, if there exists $K>0$ such that $M(2 u) \leq K M(u), u \geq 0$.

Lemma 1.1. Let $M$ be an Orlicz function which satisfies $\Delta_{2}-$ condition and let $0<\delta<1$. Then for each $t \geq \delta$, we have $M(t)<K \delta^{-1} M(2)$ for some constant $K>0$.

Recently different classes of sequences have been introduced by using an Orlicz function and their algebraic and topological properties have been investigated by Et et.al. [1], Tripathy and Mahanta [18], Tripathy et.al. [20], Tripathy and Hazarika [15], Tripathy and Sarma $[24,30]$ and many others.

A double sequence space $E$ is said to be solid or normal if $\left(\alpha_{k, l} x_{k, l}\right) \in E$, whenever $\left(x_{k, l}\right) \in E$ and for all double sequences $\alpha=\left(\alpha_{k, l}\right)$ of scalars with $\left|\alpha_{k, l}\right| \leq 1$ for all $k, l \in \mathbb{N}$.

Let $n \in \mathbb{N}$ and $X$ be a real vector space of dimension $d$, where $n \leq d$. A real-valued function $\|., \ldots,$.$\| on X$ satisfying the following four conditions:

(i) $\left\|\left(x_{1}, x_{2}, \ldots, x_{n}\right)\right\|=0$ if and only if $x_{1}, x_{2}, \ldots, x_{n}$ are linearly dependent,

(ii) $\left\|\left(x_{1}, x_{2}, \ldots, x_{n}\right)\right\|$ is invariant under permutation,

(iii) $\left\|\alpha x_{1}, x_{2}, \ldots, x_{n}\right\|=|\alpha|\left\|\left(x_{1}, x_{2}, \ldots, x_{n}\right)\right\|, \alpha \in \mathbb{R}$,

(iv) $\left\|\left(x_{1}+x_{1}^{2}, x_{2}, \ldots, x_{n}\right)\right\| \leq\left\|\left(x_{1}, x_{2}, \ldots, x_{n}\right)\right\|+\left\|\left(x_{1}^{2}, x_{2}, \ldots, x_{n}\right)\right\|$

is called an $n$-norm on $X$, and the pair $(X,\|(., \ldots,)\|$.$) is called an n$-normed space [3].

A trivial example of $n$-normed space is $X=\mathbb{R}$ equipped with the following Euclidean $n$-norm:

$$
\left\|\left(x_{1}, x_{2}, \ldots, x_{n}\right)\right\|_{E}=a b s\left(\left|\begin{array}{c}
x_{11} \ldots x_{1 n} \\
\ldots \\
x_{n 1} \ldots x_{n n}
\end{array}\right|\right)
$$

where $x_{i}=\left(x_{i 1}, \ldots, x_{i n}\right) \in \mathbb{R}^{n}$ for each $i=1,2, \ldots, n$.

The notion of $n$-norm from different aspect by Gahler [2], Gunawan and Mashadi [4], Misiak [8], Tripathy and Dutta [26] and many others.

\section{Main results}

In this section we introduce the notion of different types of $I$-convergent double sequences. This generalizes and unifies different notions of convergence for double sequences. We shall denote the ideal of $2^{\mathbb{N} \times \mathbb{N}}$ by $I_{2}$.

The notion of paranormed sequence space was studied at the initial stage by Simons [14]. Later on different classes of paranormed sequence spaces were intro- 
duced and their different properties were investigated by Maddox [7], Rath and Tripathy [11], Tripathy [17], Tripathy and Sen $[16,19]$ and many others.

Let $I_{2}$ be an ideal of $2^{\mathbb{N} \times \mathbb{N}}, M$ be an Orlicz function, $p=\left(p_{k, l}\right)$ be a bounded double sequence of strictly positive real numbers and $(X,\|(., \ldots,)\|$.$) be an n$-normed space. Further $w(n-X)$ denotes $X$-valued sequence space. Now, we define the following double sequence spaces:

$$
\begin{aligned}
& w^{I_{2}}[M, p,\|(., \ldots, .)\|]_{o}= \\
& \left\{\begin{array}{c}
x=\left(x_{k, l}\right) \in w(n-X): \forall \varepsilon>0, \\
\left.\left.(n, m) \in \mathbb{N} \times \mathbb{N}: \frac{1}{n m} \sum_{k, l=1,1}^{m, n}\left[M\left(\left\|\left(\frac{x_{k, l}}{\rho}, z_{1}, \ldots, z_{n-1}\right)\right\|\right)\right]^{p_{k, l}} \geq \varepsilon\right\} \in I_{2}\right\} \\
\text { for some } \rho>0 \text { and for every } z_{1}, \ldots, z_{n-1} \in X
\end{array}\right\} \\
& w^{I_{2}}[M, p,\|(., \ldots, .)\|]= \\
& \left\{\begin{array}{c}
\left\{\begin{array}{c}
\left\{\left(x_{k, l}\right) \in w(n-X): \forall \varepsilon>0,\right. \\
\left.(n, m) \in \mathbb{N} \times \mathbb{N}: \frac{1}{n m} \sum_{k, l=1,1}^{m, n}\left[M\left(\left\|\left(\frac{x_{k, l}-L}{\rho}, z_{1}, \ldots, z_{n-1}\right)\right\|\right)\right]^{p_{k, l}} \geq \varepsilon\right\} \in I_{2} \\
\text { for some } \rho>0, L \in X \text { and for every } z_{1}, \ldots, z_{n-1} \in X
\end{array}\right\}, \\
w^{I_{2}}[M, p,\|(., \ldots, .)\|]_{\infty}= \\
x=\left(x_{k, l}\right) \in w(n-X): \exists K>0, \\
\left\{(n, m) \in \mathbb{N} \times \mathbb{N}: \frac{1}{n m} \sum_{k, l=1,1}^{m, n}\left[M\left(\left\|\left(\frac{x_{k, l}}{\rho}, z_{1}, \ldots, z_{n-1}\right)\right\|\right)\right]^{p_{k, l}} \geq K\right\} \in I_{2} \\
\text { for some } \rho>0 \text { and for every } z_{1}, \ldots, z_{n-1} \in X
\end{array}\right.
\end{aligned}
$$

and

$$
w[M, p,\|(., \ldots, .)\|]_{\infty}=\left\{\begin{array}{c}
x=\left(x_{k, l}\right) \in w(n-X): \\
\exists K>0, \frac{1}{n m} \sum_{k, l=1,1}^{m, n}\left[M\left(\left\|\left(\frac{x_{k, l}}{\rho}, z_{1}, \ldots, z_{n-1}\right)\right\|\right)\right]^{p_{k, l}} \leq K \\
\text { for some } \rho>0 \text { and for every } z_{1}, \ldots, z_{n-1} \in X
\end{array}\right\} .
$$

If $p_{k, l}=1$ for all $k, l \in \mathbb{N}$ we obtain $w^{I_{2}}[M, p,\|., \ldots,\|]=.w^{I_{2}}[M,\|., \ldots,\|$.$] ,$ $w^{I_{2}}[M, p,\|., \ldots, .\|]_{o}=w^{I_{2}}[M,\|., \ldots, .\|]_{o}, w^{I_{2}}[M, p,\|(., \ldots, .)\|]_{\infty}=w^{I_{2}}[M,\|(., \ldots, .)\|]_{\infty}$ and $w[M, p,\|., \ldots, .\|]_{\infty}=w[M,\|., \ldots, .\|]_{\infty}$.

The following well-known inequality will be used in this study: If $0 \leq$ $\inf _{k, l} p_{k, l}=H_{o} \leq p_{k, l} \leq \sup _{k, l}=H<\infty, D=\max \left(1,2^{H-1}\right)$, then

$$
\left|x_{k, l}+y_{k, l}\right|^{p_{k, l}} \leq D\left\{\left|x_{k, l}\right|^{p_{k, l}}+\left|y_{k, l}\right|^{p_{k, l}}\right\}
$$

for all $k, l \in \mathbb{N}$ and $x_{k, l}, y_{k, l} \in \mathbb{C}$. Also $\left|x_{k, l}\right|^{p_{k, l}} \leq \max \left(1,\left|x_{k, l}\right|^{H}\right)$ for all $x_{k, l} \in \mathbb{C}$.

Theorem 2.1. The sets $w^{I_{2}}[M, p,\|(., \ldots, .)\|]_{o}, w^{I_{2}}[M, p,\|(., \ldots,)\|$.$] and$ $w^{I_{2}}[M, p,\|(., \ldots, .)\|]_{\infty}$ are linear spaces over the complex field $\mathbb{C}$. 
Proof. We will prove only for $w^{I_{2}}[M, p,\|(., \ldots, .)\|]_{o}$ and the others can be proved similarly. Let $x, y \in w^{I_{2}}[M, p,\|(., \ldots, .)\|]_{o}$ and $\alpha, \beta \in \mathbb{C}$. Then

$\left\{(n, m) \in \mathbb{N} \times \mathbb{N}: \frac{1}{n m} \sum_{k, l=1,1}^{m, n}\left[M\left(\left\|\left(\frac{x_{k, l}}{\rho_{1}}, z_{1}, \ldots, z_{n-1}\right)\right\|\right)\right]^{p_{k, l}} \geq \frac{\varepsilon}{2}\right\} \in I_{2}$, for some $\rho_{1}>0$

and

$\left\{(n, m) \in \mathbb{N} \times \mathbb{N}: \frac{1}{n m} \sum_{k, l=1,1}^{m, n}\left[M\left(\left\|\left(\frac{y_{k, l}}{\rho_{2}}, z_{1}, \ldots, z_{n-1}\right)\right\|\right)\right]^{p_{k, l}} \geq \frac{\varepsilon}{2}\right\} \in I_{2}$, for some $\rho_{2}>0$.

Since $\|., \ldots,$.$\| is a n$-norm and $M$ is an Orlicz function, the following inequality holds:

$$
\begin{aligned}
& \frac{1}{n m} \sum_{k, l=1,1}^{m, n}\left[M\left(\left\|\left(\frac{\alpha x_{k, l}+\beta y_{k, l}}{|\alpha| \rho_{1}+|\beta| \rho_{2}}, z_{1}, \ldots, z_{n-1}\right)\right\|\right)\right]^{p_{k, l}} \\
\leq & \frac{D}{n m} \sum_{k, l=1,1}^{m, n}\left[\frac{|\alpha|}{|\alpha| \rho_{1}+|\beta| \rho_{2}} M\left(\left\|\left(\frac{x_{k, l}}{\rho_{1}}, z_{1}, \ldots, z_{n-1}\right)\right\|\right)\right]^{p_{k, l}} \\
& +\frac{D}{n m} \sum_{k, l=1,1}^{m, n}\left[\frac{|\beta|}{|\alpha| \rho_{1}+|\beta| \rho_{2}} M\left(\left\|\left(\frac{y_{k, l}}{\rho_{2}}, z_{1}, \ldots, z_{n-1}\right)\right\|\right)\right]^{p_{k, l}} \\
\leq & \frac{D}{n m} \sum_{k, l=1,1}^{m, n}\left[M\left(\left\|\left(\frac{x_{k, l}}{\rho_{1}}, z_{1}, \ldots, z_{n-1}\right)\right\|\right)\right]^{p_{k, l}} \\
& +\frac{D}{n m} \sum_{k, l=1,1}^{m, n}\left[M\left(\left\|\left(\frac{y_{k, l}}{\rho_{2}}, z_{1}, \ldots, z_{n-1}\right)\right\|\right)\right]^{p_{k, l}}
\end{aligned}
$$

From the above inequality we get

$$
\begin{gathered}
\left\{(n, m) \in \mathbb{N} \times \mathbb{N}: \frac{1}{n m} \sum_{k, l=1,1}^{m, n}\left[M\left(\left\|\left(\frac{\alpha x_{k, l}+\beta y_{k, l}}{|\alpha| \rho_{1}+|\beta| \rho_{2}}, z_{1}, \ldots, z_{n-1}\right)\right\|\right)\right]^{p_{k, l}} \geq \varepsilon\right\} \\
\quad\left\{\left\{(n, m) \in \mathbb{N} \times \mathbb{N}: \frac{D}{n m} \sum_{k, l=1,1}^{m, n}\left[M\left(\left\|\left(\frac{x_{k, l}}{\rho_{1}}, z_{1}, \ldots, z_{n-1}\right)\right\|\right)\right]^{p_{k, l}} \geq \frac{\varepsilon}{2}\right\}\right. \\
\cup\left\{(n, m) \in \mathbb{N} \times \mathbb{N}: \frac{D}{n m} \sum_{k, l=1,1}^{m, n}\left[M\left(\left\|\left(\frac{y_{k, l}}{\rho_{2}}, z_{1}, \ldots, z_{n-1}\right)\right\|\right)\right]^{p_{k, l}} \geq \frac{\varepsilon}{2}\right\} .
\end{gathered}
$$

Two sets on the right hand side belong to $I_{2}$ and this completes the proof. 
It is also easy verify that the space $w[M, p,\|(., \ldots, .)\|]_{\infty}$ is also a linear space.

Theorem 2.2. For fixed $(n, m) \in \mathbb{N} \times \mathbb{N}, w[M, p,\|., \ldots, .\|]_{\infty}$ paranormed space with respect to the paranorm defined by

$g_{(n, m)}(x)=\inf \left\{\begin{array}{c}\rho^{\frac{p_{n, m}}{H}}:\left(\sup _{n, m} \frac{1}{n m} \sum_{k, l=1,1}^{m, n}\left[M\left(\left\|\left(\frac{x_{k, l}}{\rho}, z_{1}, \ldots, z_{n-1}\right)\right\|\right)\right]^{p_{k, l}}\right) \frac{1}{H} \leq 1, \\ \text { for some } \rho>0 \text { and for every } z_{1}, \ldots, z_{n-1} \in X\end{array}\right\}$.

Proof. $g_{(n, m)}(\theta)=0$ and $g_{(n, m)}(-x)=g_{(n, m)}(x)$ are easy to prove, so we omit them. Let us take $x, y \in w[M, p,\|(., \ldots, .)\|]_{\infty}$. Let

$$
A(x)=\left\{\rho>0: \sup _{n, m} \frac{1}{n m} \sum_{k, l=1,1}^{m, n}\left[M\left(\left\|\left(\frac{x_{k, l}}{\rho}, z_{1}, \ldots, z_{n-1}\right)\right\|\right)\right]^{p_{k, l}} \leq 1, \forall z \in X\right\}
$$

and

$$
A(y)=\left\{\rho>0: \sup _{n, m} \frac{1}{n m} \sum_{k, l=1,1}^{m, n}\left[M\left(\left\|\left(\frac{y_{k, l}}{\rho}, z_{1}, \ldots, z_{n-1}\right)\right\|\right)\right]^{p_{k, l}} \leq 1, \forall z \in X\right\} .
$$

Let $\rho_{1} \in A(x)$ and $\rho_{2} \in A(y)$. If $\rho=\rho_{1}+\rho_{2}$, then we have

$$
\begin{aligned}
& \sup _{n, m} \frac{1}{n m} \sum_{k, l=1,1}^{m, n}\left[M\left(\left\|\left(\frac{x_{k, l}+y_{k, l}}{\rho}, z_{1}, \ldots, z_{n-1}\right)\right\|\right)\right] \\
\leq & \frac{\rho_{1}}{\rho_{1}+\rho_{2}} \sup _{n, m} \frac{1}{n m} \sum_{k, l=1,1}^{m, n}\left[M\left(\left\|\left(\frac{x_{k, l}}{\rho_{1}}, z_{1}, \ldots, z_{n-1}\right)\right\|\right)\right] \\
& +\frac{\rho_{2}}{\rho_{1}+\rho_{2}} \sup _{n, m} \frac{1}{n m} \sum_{k, l=1,1}^{m, n}\left[M\left(\left\|\left(\frac{y_{k, l}}{\rho_{1}}, z_{1}, \ldots, z_{n-1}\right)\right\|\right)\right] .
\end{aligned}
$$

Thus

$$
\sup _{n, m} \frac{1}{n m} \sum_{k, l=1,1}^{m, n}\left[M\left(\left\|\left(\frac{x_{k, l}+y_{k, l}}{\rho_{1}+\rho_{2}}, z_{1}, \ldots, z_{n-1}\right)\right\|\right)\right]^{p_{k, l}} \leq 1
$$

and

$$
\begin{aligned}
g_{(n, m)}(x+y) & =\inf \left\{\left(\rho_{1}+\rho_{2}\right)^{\frac{p_{n, m}}{H}}: \rho_{1} \in A(x) \text { and } \rho_{2} \in A(y)\right\} \\
& \leq \inf \left\{\left(\rho_{1}\right)^{\frac{p_{n, m}}{H}}: \rho_{1} \in A(x)\right\}+\inf \left\{\left(\rho_{2}\right)^{\frac{p_{n, m}}{H}}: \rho_{2} \in A(y)\right\} \\
& =g_{(n, m)}(x)+g_{(n, m)}(y) .
\end{aligned}
$$


Now, let $\lambda_{k, l}^{u} \rightarrow \lambda$, where $\lambda_{k, l}^{u}, \lambda \in \mathbb{C}$ and $g_{(n, m)}\left(x_{k, l}^{u}-x_{k, l}\right) \rightarrow 0$ as $u \rightarrow \infty$. We have to show that $g_{(n, m)}\left(\lambda_{k, l} x_{k, l}^{u}-\lambda x_{k, l}\right) \rightarrow 0$ as $u \rightarrow \infty$. Let $A\left(x^{u}\right)=\left\{\rho_{u}>0: \sup _{n, m} \frac{1}{n m} \sum_{k, l=1,1}^{m, n}\left[M\left(\left\|\left(\frac{x_{k, l}^{u}}{\rho_{u}}, z_{1}, \ldots, z_{n-1}\right)\right\|\right)\right]^{p_{k, l}} \leq 1, \forall z \in X\right\}$

and

$A\left(x^{u}-x\right)=\left\{\rho_{u}^{\imath}>0: \sup _{n, m} \frac{1}{n m} \sum_{k, l=1,1}^{m, n}\left[M\left(\left\|\left(\frac{x_{k, l}^{u}-x_{k, l}}{\rho_{u}^{2}}, z_{1}, \ldots, z_{n-1}\right)\right\|\right)\right]^{p_{k, l}} \leq 1, \forall z \in X\right\}$.

If $\rho_{u} \in A\left(x^{u}\right)$ and $\rho_{u}^{\imath} \in A\left(x^{u}-x\right)$ then we observe that

$$
\begin{aligned}
& M\left(\left\|\left(\frac{\lambda_{k, l}^{u} x_{k, l}^{u}-\lambda x_{k, l}}{\rho_{u}\left|\lambda_{k, l}^{u}-\lambda\right|+\rho_{u}^{2}|\lambda|}, z_{1}, \ldots, z_{n-1}\right)\right\|\right) \\
\leq & M\left(\left\|\left(\frac{\lambda_{k, l}^{u} x_{k, l}^{u}-\lambda x_{k, l}^{u}}{\rho_{u}\left|\lambda_{k, l}^{u}-\lambda\right|+\rho_{u}^{2}|\lambda|}, z_{1}, \ldots, z_{n-1}\right)\right\|\right. \\
& \left.+\left\|\left(\frac{\lambda x_{k, l}^{u}-\lambda x_{k, l}}{\rho_{u}\left|\lambda_{k, l}^{u}-\lambda\right|+\rho_{u}^{u}|\lambda|}, z_{1}, \ldots, z_{n-1}\right)\right\|\right) \\
\leq & \frac{\rho_{u}\left|\lambda_{k, l}^{u}-\lambda\right|}{\rho_{u}\left|\lambda_{k, l}^{u}-\lambda\right|+\rho_{u}^{2}|\lambda|} M\left(\left\|\left(\frac{x_{k, l}^{u}}{\rho_{u}}, z_{1}, \ldots, z_{n-1}\right)\right\|\right) \\
& +\frac{\rho_{u}^{2}|\lambda|}{\rho_{u}\left|\lambda_{k, l}^{u}-\lambda\right|+\rho_{u}^{2}|\lambda|} M\left(\left\|\left(\frac{x_{k, l}^{u}-x_{k, l}}{\rho_{u}^{2}}, z_{1}, \ldots, z_{n-1}\right)\right\|\right) .
\end{aligned}
$$

From this inequality, it follows that

$$
\left[M\left(\left\|\frac{\lambda_{k, l}^{u} x_{k, l}^{u}-\lambda x_{k, l}}{\rho_{u}\left|\lambda_{k, l}^{u}-\lambda\right|+\rho_{u}^{2}|\lambda|}, z\right\|\right)\right]^{p_{k, l}} \leq 1
$$


and consequently

$$
\begin{aligned}
& g_{(n, m)}\left(\lambda_{k, l}^{u} x_{k, l}^{u}-\lambda x_{k, l}\right) \\
= & \inf \left\{\left(\rho_{u}\left|\lambda_{k, l}^{u}-\lambda\right|+\rho_{u}^{\imath}|\lambda|\right)^{\frac{p_{n, m}}{H}}: \rho_{u} \in A\left(x^{u}\right) \text { and } \rho_{u}^{\imath} \in A\left(x^{u}-x\right)\right\} \\
\leq & \left(\left|\lambda_{k, l}^{u}-\lambda\right|\right)^{\frac{p_{n, m}}{H}} \inf \left\{\left(\rho_{u}\right)^{\frac{p_{n, m}}{H}}: \rho_{u} \in A\left(x^{u}\right)\right\} \\
& +(|\lambda|)^{\frac{p_{n, m}}{H}} \inf \left\{\left(\rho_{u}^{\imath}\right)^{\frac{p_{n, m}}{H}}: \rho_{u}^{\imath} \in A\left(x^{u}-x\right)\right\} \\
\leq & \max \left\{|\lambda|,(|\lambda|)^{\frac{p_{n, m}}{H}}\right\} g_{(n, m)}\left(x_{k, l}^{u}-x_{k, l}\right) .
\end{aligned}
$$

Hence by our assumption the right hand side tends to zero as $u \rightarrow \infty$. This completes the proof.

Corollary 2.3. It can be noted that $g=\inf _{n, m \in \mathbb{N}} g_{(n, m)}$ also gives a paranorm on the above sequence spaces. However if one consider the sequence space $w[M, p,\|(., \ldots, .)\|]_{\infty}$ which is larger space than the space $w^{I_{2}}[M, p,\|., \ldots, .\|]_{\infty}$ the construction of the paranorm is not clear and we leave it as an open problem. However it should be noted that for a fixed $G \in I_{2}$, the space

$$
\begin{aligned}
& w_{G}[M, p,\|(., \ldots, .)\|]_{\infty}
\end{aligned}
$$

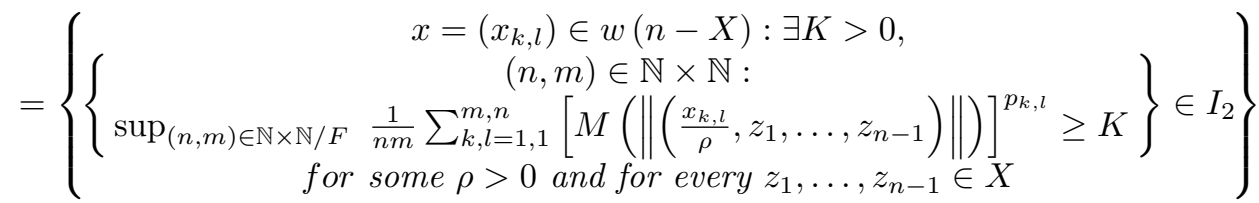

which is subspace of the space $w^{I_{2}}[M, p,\|(., \ldots, .)\|]_{\infty}$ is a paranormed space with the paranorms $g_{(n, m)}$ for $(n, m) \notin G$ and $g_{G}=\inf _{(n, m) \in \mathbb{N} \times \mathbb{N} / G} g_{(n, m)}$.

Theorem 2.4. Let $M, M_{1}$ and $M_{2}$ be Orlicz functions. Then we have

(i) $w^{I_{2}}\left[M_{1}, p,\|(., \ldots, .)\|\right]_{o} \subset w^{I_{2}}\left[M \circ M_{1}, p,\|(., \ldots, .)\|\right]_{o}$ provided that $p=$ $\left(p_{k, l}\right)$ is such that $H_{o}>0$.

(ii) $w^{I_{2}}\left[M_{1}, p,\|(., \ldots, .)\|\right]_{o} \cap w^{I_{2}}\left[M_{2}, p,\|(., \ldots, .)\|\right]_{o} \subset w^{I_{2}}\left[M_{1}+M_{2}, p,\|(., \ldots, .)\|\right]_{o}$.

Proof. (i). Let $\inf _{k, l} p_{k, l}=H_{o}$. For given $\varepsilon>0$, we first choose $\varepsilon_{o}>0$ such that $\max \left\{\varepsilon_{o}^{H}, \varepsilon_{o}^{H_{o}}\right\}<\varepsilon$. Now using the continuity of $M$, choose $0<\delta<1$ such that $0<t<\delta$ implies $M(t)<\varepsilon_{o}$. Let $x \in w^{I_{2}}\left[M_{1}, p,\|(., \ldots, .)\|\right]_{o}$. Now from the definition of the space $w^{I_{2}}\left[M_{1}, p,\|(., \ldots, .)\|\right]_{o}$, for some $\rho>0$

$$
A(\delta)=\left\{(n, m) \in \mathbb{N} \times \mathbb{N}: \frac{1}{n m} \sum_{k, l=1,1}^{m, n}\left[M_{1}\left(\left\|\left(\frac{x_{k, l}}{\rho}, z_{1}, \ldots, z_{n-1}\right)\right\|\right)\right]^{p_{k, l}} \geq \delta^{H}\right\} \in I_{2}
$$


Thus if $(n, m) \notin A(\delta)$ then

$$
\begin{aligned}
& \frac{1}{n m} \sum_{k, l=1,1}^{m, n}\left[M_{1}\left(\left\|\left(\frac{x_{k, l}}{\rho}, z_{1}, \ldots, z_{n-1}\right)\right\|\right)\right]^{p_{k, l}}<\delta^{H} \\
\Rightarrow & \sum_{k, l=1,1}^{m, n}\left[M_{1}\left(\left\|\left(\frac{x_{k, l}}{\rho}, z_{1}, \ldots, z_{n-1}\right)\right\|\right)\right]^{p_{k, l}}<n m \delta^{H}, \\
\Rightarrow & {\left[M_{1}\left(\left\|\left(\frac{x_{k, l}}{\rho}, z_{1}, \ldots, z_{n-1}\right)\right\|\right)\right]^{p_{k, l}}<\delta^{H} \text { for all } k, l=1,2, \ldots, } \\
\Rightarrow & M_{1}\left(\left\|\left(\frac{x_{k, l}}{\rho}, z_{1}, \ldots, z_{n-1}\right)\right\|\right)<\delta \text { for all } k, l=1,2, \ldots
\end{aligned}
$$

Hence from above inequality and using continuity of $M$, we must have

$$
M\left(M_{1}\left(\left\|\left(\frac{x_{k, l}}{\rho}, z_{1}, \ldots, z_{n-1}\right)\right\|\right)\right)<\varepsilon_{o} \text { for all } k, l=1,2, \ldots
$$

which consequently implies that

$$
\begin{aligned}
& \sum_{k, l=1,1}^{m, n}\left[M\left(M_{1}\left(\left\|\left(\frac{x_{k, l}}{\rho}, z_{1}, \ldots, z_{n-1}\right)\right\|\right)\right)\right]^{p_{k, l}}<n m \max \left\{\varepsilon_{o}^{H}, \varepsilon_{o}^{H_{o}}\right\}<n m \varepsilon, \\
\Rightarrow & \frac{1}{n m} \sum_{k, l=1,1}^{m, n}\left[M\left(M_{1}\left(\left\|\left(\frac{x_{k, l}}{\rho}, z_{1}, \ldots, z_{n-1}\right)\right\|\right)\right)\right]^{p_{k, l}}<\varepsilon .
\end{aligned}
$$

This shows that

$$
\left\{(n, m) \in \mathbb{N} \times \mathbb{N}: \frac{1}{n m} \sum_{k, l=1,1}^{m, n}\left[M\left(M_{1}\left(\left\|\left(\frac{x_{k, l}}{\rho}, z_{1}, \ldots, z_{n-1}\right)\right\|\right)\right)\right]^{p_{k, l}} \geq \varepsilon\right\} \subset A(\delta)
$$

and so belongs to $I_{2}$. This completes the proof. fact that

(ii) Let $x \in w^{I_{2}}\left[M_{1}, p,\|(., \ldots, .)\|\right]_{o} \cap w^{I_{2}}\left[M_{2}, p,\|(., \ldots, .)\|\right]_{o}$. Then the

$$
\begin{aligned}
& \frac{1}{n m}\left[\left(M_{1}+M_{2}\right)\left(\left\|\left(\frac{x_{k, l}}{\rho}, z_{1}, \ldots, z_{n-1}\right)\right\|\right)\right]^{p_{k, l}} \\
\leq & \frac{D}{n m}\left[M_{1}\left(\left\|\left(\frac{x_{k, l}}{\rho}, z_{1}, \ldots, z_{n-1}\right)\right\|\right)\right]^{p_{k, l}}+\frac{D}{n m}\left[M_{2}\left(\left\|\left(\frac{x_{k, l}}{\rho}, z_{1}, \ldots, z_{n-1}\right)\right\|\right)\right]^{p_{k, l}}
\end{aligned}
$$

gives us the result.

Theorem 2.5. (i) If $0<\inf _{k, l} p_{k, l}=H_{o} \leq p_{k, l}<1$, then $w^{I_{2}}[M, p,\|(., \ldots, .)\|]_{o} \subset$ $w^{I_{2}}[M,\|(., \ldots, .)\|]_{o}$. 
(ii) If $1 \leq p_{k, l} \leq \sup _{k, l} p_{k, l}=H<\infty$, then $w^{I_{2}}[M,\|(., \ldots, .)\|]_{o} \subset$ $w^{I_{2}}[M, p,\|(., \ldots, .)\|]_{o}$.

(iii) If $0<p_{k, l}<q_{k, l}<\infty$ and $\frac{q_{k, l}}{p_{k, l}}$ is bounded, then $w^{I_{2}}[M, p,\|., \ldots, .\|]_{o} \subset$ $w^{I_{2}}[M, q,\|(., \ldots, .)\|]_{o}$.

Proof. The proof is standard, so we omit it.

Theorem 2.6. The sequence spaces $w^{I_{2}}[M, p,\|(., \ldots, .)\|]_{o}, w^{I_{2}}[M, p,\|(., \ldots,)\|$.$] ,$ $w^{I_{2}}[M, p,\|(., \ldots, .)\|]_{\infty}$ and $w[M, p,\|., \ldots, .\|]_{\infty}$ are solid.

Proof. We give the proof for only $w^{I_{2}}[M, p,\|(., \ldots, .)\|]_{o}$. The others can be proved similarly. Let $x \in w^{I_{2}}\left[M_{1}, p,\|(., \ldots, .)\|\right]_{o}$ and $\alpha=\left(\alpha_{k, l}\right)$ be a double sequence of scalars such that $\left|\alpha_{k, l}\right| \leq 1$ for all $k, l \in \mathbb{N}$. Then we have

$$
\begin{aligned}
& \left\{(n, m) \in \mathbb{N} \times \mathbb{N}: \frac{1}{n m} \sum_{k, l=1,1}^{m, n}\left[M\left(\left\|\left(\frac{\alpha_{k, l} x_{k, l}}{\rho}, z_{1}, \ldots, z_{n-1}\right)\right\|\right)\right]^{p_{k, l}} \leq \varepsilon\right\} \\
\subset & \left\{(n, m) \in \mathbb{N} \times \mathbb{N}: \frac{T}{n m} \sum_{k, l=1,1}^{m, n}\left[M\left(\left\|\left(\frac{x_{k, l}}{\rho}, z_{1}, \ldots, z_{n-1}\right)\right\|\right)\right]^{p_{k, l}} \leq \varepsilon\right\} \in I_{2},
\end{aligned}
$$

where $T=\max _{k, l}\left\{1,\left|\alpha_{k, l}\right|^{H}\right\}$. Hence $\alpha x \in w^{I_{2}}\left[M_{1}, p,\|(., \ldots, .)\|\right]_{o}$ for all double sequences $\alpha=\left(\alpha_{k, l}\right)$ with $\left|\alpha_{k, l}\right| \leq 1$ for all $k, l \in \mathbb{N}$ whenever $x \in$ $w^{I_{2}}\left[M_{1}, p,\|(., \ldots, .)\|\right]_{o}$.

\section{References}

[1] M. Et, Y. Altin, B. Choudhary and B. C. Tripathy, On some classes of sequences defined by sequences of Orlicz functions, Mathematical Inequalities and Applications, $\mathbf{9}(2)(2006), 335-342$.

[2] S. Gahler, Linear 2-normietre Rume, Math.Nachr., 28(1965), 1-43.

[3] H. Gunawan, On n-inner product, n-norms and the Cauchy-Schwarz Inequality, Scientiae Mathematicae Japonicae Online, 5(2001), 47-54.

[4] H. Gunawan and M. Mashadi, On n-normed spaces, Int. J. Math. \& Math. Sci., 27(10)(2001), 631-639.

[5] P. Kostyrko, T. Salat and W. Wilczynski,I-convergence, Real Analysis Exchange, 26(2)(2000/2001), 669-686.

[6] M. A. Krasnoselski and Y. B. Rutickii, Convex function and Orlicz spaces, Groningen, Nederland, 1961.

[7] I. J. Maddox, Paranormed sequence spaces generated by infinite matrices, Proc. Camb. Phil. Soc., 64(1968), 285-290. 
[8] A. Misiak, n-inner product spaces, Math. Nachr., 140(1989), 299-319.

[9] H. Nakano, Concave modulars, J. Math. Soc. Japan, 5(1953), 29-49.

[10] A. Pringsheim, Zur Theori der zweifach unendlichen Zahlenfolgen, Math. Ann. 53(1900), 289-321.

[11] D. Rath and B. C. Tripathy, Matrix maps on sequence spaces associated with sets of integers, Indian Jour. Pure Appl. Math., 27(2)(1996), 197-206.

[12] W. H. Ruckle, FK-spaces in which the sequence of coordinate vectors is bounded, Canad. J. Math., 25(1973), 973-978.

[13] T. Salat, B. C. Tripathy and M. Ziman, On I-convergence field, Italian J. Pure and Appl. Math., 17(2005), 45-54.

[14] S. Simons, The sequence space $l\left(p_{v}\right)$ and $m\left(p_{v}\right)$, Proc. London Math. Soc., 15(3)(1965), 422-436.

[15] B. C. Tripathy and B. Hazarika, I-convergent sequence spaces defined by Orlicz function, Acta Mathematica Applicatae Sinica, 27(1)(2001), 149-154.

[16] B. C. Tripathy and M. Sen, On generalized statistically convergent sequences, Indian Jour. Pure Appl. Math., 32(11)(2001), 1689-1694.

[17] B. C. Tripathy, On generalized difference paranormed statistically convergent sequences, Indian Jour. Pure Appl. Math., 35(5)(2004), 655-663.

[18] B. C. Tripathy and S. Mahanta, On a class of generalized lacunary difference sequence spaces defined by Orlicz function, Acta Mathematica Applicatae Sinica, (Eng.Ser.), $\mathbf{2 0}(2)(2004), 231-238$.

[19] B. C. Tripathy and M. Sen, Characterization of some matrix classes involving paranormed sequence spaces, Tamkang Journal of Mathematics, 37(2)(2006), 155-162.

[20] B. C. Tripathy, Y. Altin and M. Et, Generalized difference sequence spaces on seminormed spaces defined by Orlicz functions, Mathematica Slovaca, 58(3)(2008), 315324 .

[21] B. C. Tripathy and B. Hazarika, I-convergent sequence spaces associated with multiplier sequence spaces, Mathematical Inequalities and Applications, 11(3)(2008), 543548.

[22] B. C. Tripathy and B. Sarma, Statistically convergent difference double sequence spaces, Acta Mathematica Sinica, 24(5)(2008), 737-742.

[23] B. C. Tripathy and B. Hazarika, Paranormed I-convergent sequence spaces, Mathematica Slovaca, 59(4)(2009), 485-494.

[24] B. C. Tripathy and B. Sarma, Vector valued double sequence spaces defined by Orlicz function, Mathematica Slovaca, 59(6)(2009), 767-776.

[25] B. C. Tripathy and A. J. Dutta, Bounded variation double sequence space of fuzzy real numbers, Computers \& Mathematics with Applications, 59(2)(2010), 1031-1037.

[26] B. C. Tripathy and H. Dutta, On some new paranormed difference sequence spaces defined by Orlicz functions, Kyungpook Mathematical Journal, 50(2010), 59-69.

[27] B. C. Tripathy and S. Mahanta, On I-acceleration convergence of sequences, Journal of the Franklin Institute, 347(2010), 591-598. 
[28] B. C. Tripathy and P. Chandra, On some generalized paranormed sequence spaces associated with multiplier sequences defined by modulus function, Analysis in Theory and Applications, 27(1)(2011), 21-27.

[29] B. C. Tripathy and B. Hazarika, I-monotonic and I-convergent sequences, Kyungpook Mathematical Journal, 51(2)(2011), 233-239.

[30] B. C. Tripathy and B. Sarma, Double sequence spaces of fuzzy numbers defined by Orlicz function, Acta Mathematica Sinica, 31B(1)(2011), 134-140. 